PSYCHOMETRIKA-VOL. 26, NO. 2

JUNE, 1961

\title{
A DOUBLE LAW OF COMPARATIVE JUDGMENT FOR THE ANALYSIS OF PREFERENTIAL CHOICE AND SIMILARITIES DATA*
}

\author{
C. H. Coombs, M. Greenberg, and J. Zrnnest
}

UNIVERSITY OF MICHIGAN

\begin{abstract}
By virtue of certain modifications in the Law of Comparative Judgment, equations are developed which $(i)$ permit the construction of a joint scale of individuals and items, as in the case of attitude measurement, directly from their pair-comparison preferences, and $(i i)$ take into account the variable of laterality which is significant for the construction of group preference scales.
\end{abstract}

This paper is concerned with the theoretical implications that the unfolding model of preferential choice [1] has for the Law of Comparative Judgment [4]. By the unfolding model, each individual and stimulus is viewed in terms of a distribution of "discriminal processes" located in the same space. On each pair-comparison trial the individual is represented by a point from his distribution and each of the two stimuli by points from their respective distributions. The individual's preferential choice on a given trial is assumed to reffect which of the two stimulus points is nearer the individual's point on that trial.

That this theory has implications for the application of the Law of Comparative Judgment to pairwise preferential choice data has already been suggested by experimental results [3]. This experiment indicates that if the distributions of discriminal processes of the stimuli are both on the same side of, or unilateral to, the individual's distribution, the inconsistency of judgment is of a different order of magnitude than if the distributions are on opposite sides (bilateral). The consequence of this, in brief, is that the usual data matrix containing the proportion of times stimulus $j$ has been preferred to stimulus $k$ should be partitioned into two distinct data matrices. One matrix has proportions in each cell based on that subset of individuals for whom that pair of stimuli is unilateral, and the other matrix has proportions based on the subjects for whom that pair of stimuli is bilateral.

Because the relation of inconsistency to psychological distance is of a different order of magnitude for these two matrices, the Law of Comparative Judgment for each is different and hence gives rise to the reference in the title to a double Law of Comparative Judgment, a unilateral law for the unilateral matrix, and a bilateral law for the other. In the following section

*This work was supported by grant NSF-G5820 from the National Science Foundation. †Now at Stanford University. 
the two laws are developed in order to show the theoretical implications of the unfolding theory for the Law of Comparative Judgment, and in the final section some of the implications for and difficulties of practical applications are pointed out.

\section{Unilateral and Bilateral Equations}

The unfolding theory of preferential choice postulates the existence of a space consisting of ideal points for individuals, denoted by $c$ 's, and points corresponding to stimuli, denoted by $q$ 's. Throughout the remainder of this paper it will be assumed that this space is one-dimensional, and it will be called a $J$ or joint scale. The algebraic distance from the ideal point of individual $i$ to the stimulus $j$, at the moment $h$, is then defined as

$$
p_{h i j}=c_{h i j}-q_{h i j} .
$$

In terms of this model, individual $i$ will prefer stimulus $j$ to stimulus $k$ at the moment $h$ if and only if

$$
\left|p_{h i j}\right|-\left|p_{h i k}\right| \leq 0 .
$$

Alternatively, the preferential choice of an individual at a given moment signifies which stimulus point is nearer his ideal point. Furthermore, the percentage of times that one observes stimulus $j$ preferred to stimulus $k$, then, is the percentage of times that (2) obtains. From (1)

$$
\left|p_{h i}\right|-\left|p_{h i k}\right|=\left|c_{h i}-q_{h i i}\right|-\left|c_{h i k}-q_{h i k}\right| \text {. }
$$

It is assumed in the following discussion that when an individual is judging a pair of stimuli at a given moment, only one ideal point is involved. Thus, for stimuli $j$ and $k$

$$
c_{h i i}=c_{h i k}=c_{h i} .
$$

The development of these equations may be pursued in the context of case I of the Law of Comparative Judgment (replications on a single individual) or case II (replications over individuals). Because the possible applications of these developments will more likely be in the context of case II, the assumption will be made in what follows that each of a number of individuals has responded once to every pair of stimuli. Adaptations to case I or to a combination of case I and II, in which each of a number of individuals responds a number of times to each pair, are relatively straightforward and add little to the theoretical implications from case II alone. Hence in what follows the subscript $h$ will be dropped so that individually replicated judgments will not be explicitly considered.

It is necessary to distinguish between those pairs of stimuli which are unilateral with respect to a given individual and those pairs which are bilateral. A stimulus pair $(j, k)$ is unilateral to the subject located at $c_{\mathrm{t}}$ if both 
stimuli have scale values, $q_{i i}$ and $q_{i k}$, less than or greater than $c_{i}$. More simply, both stimuli lie on the same side of $c_{i}$. For a bilateral stimulus pair, the two stimuli are on opposite sides of $c_{i}$. Formally, stimuli $j$ and $k$ are unilateral to $c_{i}$ if and only if

$$
\left(q_{i i} \lessgtr c_{i}\right) \Leftrightarrow\left(q_{i k} \lessgtr c_{i}\right) .
$$

Stimuli $j$ and $k$ are bilateral to $c_{i}$ if and only if

$$
\left(q_{i j} \lessgtr c_{i}\right) \Leftrightarrow\left(q_{i k} \gtrless c_{i}\right) \text {. }
$$

The unilateral equations will be developed first. As is evident from (5), if $q_{i j}<c_{i}$ and $q_{i k}<c_{i}$ then the individual is to the right of both stimuli; this is called condition $R$. (If $q_{i j}>c_{i}$ and $q_{i k}>c_{i}$ then we have condition $L_{i}$ )

Consider first condition $L$. From (5), since $c_{i}$ is less than $q_{i j}$ and $q_{i k}$, (3) reduces to

$$
\left|p_{i j}\right|^{L}-\left|p_{i k}\right|^{L}=\left(q_{i i}-c_{i}^{L}\right)-\left(q_{i k}-c_{i}^{L}\right)=q_{i j}-q_{i k},
$$

and similarly, for $c_{i}$ to the right of this unilateral pair,

$$
\left|p_{i j}\right|^{R}-\left|p_{i k}\right|^{R}=q_{i k}-q_{i i} \text {. }
$$

Equations (7) and (8) indicate that the preferential choice of an individual for one of two unilateral stimuli is mediated by the difference between the scale values of the stimuli on the joint scale. This immediately suggests that the preferential choices of those individuals unilateral to a pair of stimuli can be used to scale the stimuli on the joint scale.

To simplify matters, the well-known case $V$ assumptions will be made, i.e., that the stimuli project normal distributions on the $J$ scale with equal variances,

$$
q_{i i} \text { is } N\left(Q_{i}, \sigma_{q}^{2}\right) \text {, }
$$

and that the correlation, over individuals, between each pair of stimuli is a constant,

$$
r_{i} a_{i q i k}=\underset{i}{r_{g q}} \text { for all pairs } j, k \text {. }
$$

The unilateral Law of Comparative Judgment may then be written as follows:

$$
\left|P_{i}\right|^{L}-\left|P_{k}\right|^{L}=X_{k i}^{L} \sigma_{a} \sqrt{2\left(1-r_{Q q}\right)}=Q_{i}-Q_{k},
$$

where $X_{k i}^{L}$ denotes the normal deviate corresponding to the proportion of unilateral-left persons preferring stimulus $j$ to $k$ and

$$
\left|P_{i}\right|^{L}=\underset{i}{E}\left|p_{i i}\right|^{L} \text {. }
$$

There is, of course, an equivalent expression which may be written for the $R$ condition but, as will be discussed in the next section, only one of these is necessary in application. 
The development of the Law of Comparative Judgment equation for the bilateral case parallels the unilateral treatment. As is evident from (6), if $q_{i i}<c_{i}<q_{i k}$ or if $q_{i j}>c_{i}>q_{i k}$ then the individual is between the stimuli on the joint scale and the stimuli are bilateral to the individual, in which case (3) can be written as follows (one may assume $q_{i j}<c_{i}<q_{i k}$ without any loss of generality):

$$
\begin{aligned}
\left|p_{i j}\right|^{R}-\left|p_{i k}\right|^{L} & =\left(c_{i}^{R_{j} L_{k}}-q_{i j}\right)-\left(q_{i k}-c_{i}^{R_{j} L_{k}}\right) \\
& =2 c_{i}^{R_{i} L_{k}}-q_{i j}-q_{i k},
\end{aligned}
$$

where $c_{i}^{R i L k}$ denotes the $c_{i}$ of those individuals who are to the right of stimulus $j$ and to the left of stimulus $k$.

A comparison of (12) with (7) or (8) makes evident the source of the essential difference between unilateral and bilateral preference judgments. In the unilateral case preference is mediated by the difference between the two scale values of the stimuli, completely independent of the $c_{i}$ 's. In the bilateral case, on the other hand, the $c_{i}$ 's enter in a significant way, and in particular, it is evident that the variance of the differences $\left|p_{i j}\right|^{R}-\left|p_{i k}\right|^{2}$ includes among its components the variance of the $c_{i}^{R_{i} L_{k}}$.

To simplify a good deal of tedious algebra, one may make the same case $V$ assumptions previously introduced into the unilateral case [see (9) and (10)] and in addition assume

$$
r_{c i q_{i j}}=r_{c i q i k}=r_{c q} \text {. }
$$

The variance of the differences on the left-hand side of (12), called the bilateral comparatal variance, may be written

$$
\sigma_{j k}^{2}=4 \sigma_{c}^{2}-8 r_{c \varepsilon} \sigma_{c} \sigma_{a}+2 \sigma_{a}^{2}\left(1+r_{a q}\right),
$$

where $\sigma_{c}^{2}$ is the variance of the $c_{i}$ which are bilateral to the pair of stimuli $j$ and $k$.

The bilateral Law of Comparative Judgment with case $\mathrm{V}$ assumptions may then be written as

$$
\left|P_{i}\right|^{R}-\left|P_{k}\right|^{L}=X_{k i}^{B} \sqrt{4 \sigma_{c}^{2}-8 r_{c q} \sigma_{c} \sigma_{q}+2 \sigma_{a}^{2}\left(1+r_{q q}\right)} .
$$

This bilateral comparatal variance is distinctly different from the unilateral comparatal variance under the same assumptions since, from (11), it is evident that the unilateral comparatal variance, $\sigma_{u}^{2}$, is

$$
\sigma_{u}^{2}=2 \sigma_{a}^{2}\left(1-r_{a q}\right) .
$$

If $\sigma_{u}^{2}$ is set equal to one for the unit of measurement in the unilateral case the bilateral comparatal variance may have some value quite different from one. The bilateral pairwise percentages are generated not only on the basis of a different unit of measurement, but, as may be seen from (12), are 
estimates of a different variable than unilateral pairwise percentages. Unilateral and bilateral pairwise preferential choices should therefore not be combined in the same probability matrix and analyzed by the Law of Comparative Judgment.

\section{Applications}

Equations (11) and (14), for unilateral and bilateral judgments, respectively, constitute what is here called the double Law of Comparative Judgment. It is clear from these equations that according to the unfolding model of preferential choice the inconsistency measure for unilateral and bilateral pairs of stimuli must be differently translated into psychological distance, and furthermore, the inconsistency measures represent different variables.

There are two practical consequences. One is the possibility that arises for constructing the joint scale (i.e., the $C_{i}$ and $Q_{i}$ values) directly from preferential choice data instead of the usual two-step procedure of scaling the stimuli first and then getting preferential choice data. The second consequence is a revised procedure for translating the pairwise probabilities from similarities data into measures of distance. Both of these practical consequences are discussed in order in more detail below.

Any application of this development requires an initial step: knowing the approximate order of the stimuli on the $J$ scale. If this order is not known from a priori considerations it can be obtained by utilizing the unfolding technique, which would also provide the approximate locations of the subjects with respect to the stimuli. The most serious problems in locating individuals from inconsistent data tend to arise with individuals centrally located on the $J$ scale, i.e., who have a maximum number of bilateral pairs of stimuli.

This is fortunate for constructing the $J$ scale in that it is the unilateral Law of Comparative Judgment which is needed for that purpose and it uses data only from individuals unilateral to a pair of stimuli. The entries in the unilateral matrix are obtained by the following procedure. There are $N^{L}$ individuals to the left of stimuli $j$ and $k$ of whom $n_{j k}^{L}$ prefer $j$ to $k$. Similarly there are $N^{R}$ individuals to the right of stimuli $j$ and $k$ of whom $n_{k j}^{R}$ prefer $k$ to $j$. A combined estimate of the proportion of individuals who judge $k$ to be greater than $j$ is

$$
\frac{n_{i k}^{L}+n_{k i}^{R}}{\overline{N^{L}+N^{R}}}
$$

If $X_{k}$ represents the normal deviate corresponding to the proportion in (16), it is clear from (11) that

$$
Q_{k}-Q_{i}=X_{k i} \sigma_{q} \sqrt{2\left(1-r_{q q}\right)} .
$$


Thus case $V$ of the unilateral Law of Comparative Judgment may be applied to scale the stimuli on the $J$ scale, and each individual may be assigned to an interval corresponding to his preference ordering.

The second consequence of this development is concerned with the analysis of similaritics data to scale the distances between pairs of stimuli. In the first place it must be evident that this model of preferential choice has certain characteristics in common with similarities data. An individual making a preferential choice is, according to this model, judging the relative similarity of the stimuli to a hypothetical ideal point, so his judgment reflects an order relation on a pair of distances. One sometimes scales these distances from preferential choice data in order to construct a scale of the stimuli from most to least preferred. One scales the distances from similarities data in order to apply a multidimensional psychophysical model [5]. The double Law of Comparative Judgment has implications for both of these kinds of data.

Just the construction of a preferability scale of the stimuli (i.e., the $P_{i}$ values) based on the entire set of subjects will be discussed. This has generally been done by applying the Law of Comparative Judgment to the proportion of times the members of a group have preferred each stimulus to every other, without any distinction between individuals. However (14) calls this procedure into question because the bilateral comparatal variance of each pair of stimuli is a function, among other things, of the variance of those individuals' ideal points for whom that pair is a bilateral pair. This value will in general be different for every pair of stimuli. A solution involves some difficult estimation problems and/or strong assumptions. For example one might assume $r_{c q}=r_{q q}=0$, letting $\sigma_{c}=m \sigma_{q}$, then (14) becomes

$$
P_{i}^{R}-P_{k}^{L}=X_{k j}^{B} \sigma_{a} \sqrt{2} \sqrt{2 m^{2}+1} \text {. }
$$

Letting $\sigma_{0} \sqrt{2}=1$ for the unit of measurement in the unilateral case, a solution to (18) is possible for the matrix of bilateral data only if the parameter $m$ is known. At present only crude methods are available for estimating it, and none is recommended.

The final solution then for the group scale of preferability would involve a weighted average of the solutions to (18), (11) based only on unilateral left cases, and the corresponding equation for unilateral right cases. This procedure is recommended only in the absence of any better alternative and serves primarily to indicate how very different the problem is from that assumed in the conventional procedure.

Another area of application of these methods that is most promising is the area of similarities data. The frequency with which an individual judges stimulus $B$ or $C$ to be most like $A$ is formally equivalent to $A$ 's preferential choice for the nearer one. In this case, if a one-dimensional scale may be obtained by the unfolding technique [2], then the unilateral law is appli- 
cable for constructing an interval scale. If one wishes to scale the distances between pairs of stimuli then both laws need to be applied, and in the bilateral case, under case $V$ assumptions, $m=1$, because the variance of the $c$ values between two stimuli is itself the variance of a stimulus. In this case (18) becomes

$$
\left|P_{i}\right|^{R}-\left|P_{k}\right|^{L}=X_{k j}^{B} \sigma_{a} \sqrt{2} \sqrt{3}
$$

So if $\sigma_{Q} \sqrt{2}$ is set equal to 1 for the unit of measurement for the unilateral matrix, whereas $\sigma_{a} \sqrt{2} \sqrt{3}$ is used for the same purpose for the bilateral matrix of proportions, then the $X_{k j}^{B}$ values from the bilateral matrix must be multiplied by $\sqrt{3}$ before combining with the $X_{k}$ from the unilateral matrix to form a weighted average. This is because the unit of measurement used for the bilateral percentages is $\sqrt{3}$ times as large as the unit used for converting the unilateral percentages. This is only true when the stimuli lie on a one-dimensional scale. The generalization of the effect of laterality on the comparatal variance for stimuli in a multidimensional space, while simple in principle, presents estimation problems which have not yet been solved.

A theoretical analysis of pairwise preferential choices is made in the spirit of the Law of Comparative Judgment but from the point of view of the unfolding theory of preferential choice behavior. The analysis reveals that for every pair of stimuli, the subjects must be partitioned into those who are (i) to the left of both stimuli on the $J$ scale, (ii) between them, and (iii) to the right of both. The comparatal variance is seen to be different for (ii) than for (i) and (iii). It is shown how partitioning of the $S$ s will permit construction of a $J$ scale directly from the preferential choices but a group scale of preferability has no simple solution. The appropriateness of this development for similarities data as well as preferential choice is pointed out.

\section{REFERENCES}

[1] Coombs, C. H. A theory of psychological scaling. Engng Res. Bull. No. 34, Ann Arbor, Mich.: Univ. Michigan Press, 1952.

[2] Coombs, C. H. A method for the study of interstimulus similarity. Psychometrika, 1954, 19, 183-194.

[3] Coombs, C. H. On the use of inconsistency of preferences in psychological measurement. J. exp. Psychol., 1958, 55, 1-7.

[4] Thurstone, L. L. The measurement of values. Chicago: Univ. Chicago Press, 1959.

[5] Torgerson, W. S. Theory and methods of scaling. New York: Wiley, 1958.

\section{Manuscript received 8/10/59}

Revised manuscript received $\$ / 28 / 60$ 